\title{
Interaction/merger-induced starbursts in local very metal-poor dwarfs: link to the common SF in high- $z$ young galaxies
}

\author{
S. A. Pustilnik ${ }^{1}, \mathbf{E k t a}^{2}$, A. Y. Kniazev ${ }^{3}$, J. N. Chengalur ${ }^{2}$ \\ and L. Vanzi ${ }^{4}$ \\ ${ }^{1}$ Special Astrophysical Obs. RAS, Nizhnij Arkhyz, 369167, Russia email: sap@sao.ru \\ ${ }^{2}$ National Centre of Radio Astrophysics, Pune 411007, India email: ekta@ncra.tifr.res.in \\ ${ }^{3}$ South African Astronomical Obs. Cape Town 7935, South Africa email: akniazev@saao.ac.za \\ ${ }^{4}$ Europian Southern Obs. Santiago, Chile email: lvanzi@eso.org
}

\begin{abstract}
We present a subsample of 'local' very metal-poor gas-rich galaxies that show more or less clear evidences of interactions and mergers and discuss their relevance to the study of high-redshift star-forming young galaxies.
\end{abstract}

Keywords. galaxy abundances, galaxy interactions, star formation

\section{Introduction}

Widespread galaxy formation from pregalactic gas took place in the first 1-3 Gyr after the Big Bang, with most of them forming in low-mass halos (i.e., $\mathrm{M}$ in the range of $\left.10^{7}-10^{10} \mathrm{M}_{\odot}\right)$. Observations at high redshifts $(z=4-7)$ are, however, mainly limited to the rare massive "tip of the iceberg" objects. Detailed studies of the properties and the evolution of more common lower-mass young galaxies will have to await the next generation mega telescopes. Their local analogs provide clues for their SF and evolution.

\section{Method and Results}

We have conducted a multi-wavelength (including optical/NIR morphology/photometry, $\mathrm{HI}$ imaging, and $\mathrm{H} \alpha$-line kinematics) study of a sample of the local eXtremely Metal-Deficient (XMD) BCGs and find in a large fraction of them clear evidences that strong interactions or mergers with low-mass objects, provide a trigger mechanism for their observed starbursts. We present about 20 such XMD BCGs arranged in a Toomrelike sequence and also the first results of detailed studies of several individual objects.

\section{Conclusions}

Since both SF (through cooling rate and the IMF) and its feedback (through the massive star evolution and interaction with the ISM) depend substantially on the ISM metallicity, comprehensive multiwavelength studies of local XMD galaxy mergers, coupled with theoretical modelling, should give us substantial insight into star formation in young high-redshift galaxies.

\section{Acknowledgements}

S. A. P. would like to acknowledge the support from IAU (grant No.12330) and Czech Academy of Sciences. 